\title{
Supermassive Black Holes, Large Scale Structure and Holography
}

\author{
T. R. Mongan \\ 84 Marin Avenue, Sausalito, USA \\ Email: tmongan@gmail.com
}

Received March 21, 2013; revised April 29, 2013; accepted June 7, 2013

Copyright (C) 2013 T. R. Mongan. This is an open access article distributed under the Creative Commons Attribution License, which permits unrestricted use, distribution, and reproduction in any medium, provided the original work is properly cited.

\begin{abstract}
A holographic analysis of large scale structure in the universe provides an upper bound on the mass of supermassive black holes at the center of large scale structures with matter density varying as $\frac{1}{r^{2}}$ as a function of distance $r$ from their center. The upper bound is consistent with two important test cases involving observations of the supermassive black hole with mass $3.6 \times 10^{-6}$ times the galactic mass in Sagittarius $A^{*}$ near the center of our Milky Way and the $2 \times$ $10^{9}$ solar mass black hole in the quasar ULAS J112001.48+064124.3 at redshift $z=7.085$. It is also consistent with upper bounds on central black hole masses in globular clusters M15, M19 and M22 developed using the Jansky Very Large Array in New Mexico.
\end{abstract}

Keywords: Supermassive Black Holes; Large Scale Structure; Holographic Principle

\section{Introduction}

How supermassive black holes “... form and evolve inside galaxies is one of the most fascinating mysteries in modern astrophysics" [1]. This analysis addresses that issue with a holographic model [2] for large scale structure in the universe, based on the holographic principle [3] resulting from the theory of gravitation expressed by general relativity. The internal dynamics of large scale structures is analyzed using classical Newtonian gravity to describe the motion of sub-elements within the structures and general relativity to describe the supermassive black holes at their centers. Consistency of the results with test cases across the range of large scale structures and redshifts makes it difficult to ascribe those results to numerical coincidences.

\section{Internal Dynamics of Large Scale Structures}

The holographic model for large scale structure [2] identifies three levels of self-similar large scale structures (corresponding to superclusters, galaxies and star clusters) between stellar systems and the totality of today's observable universe. The extended holographic principle employed in that model indicates all information describing physics of a gravitationally-bound astronomical system of total mass $M_{s}$ is encoded on a spherical holographic screen enclosing the system. In our vacuum-dominated universe, the radius of the holographic screen encoding all information describing a structure of mass $M_{s}$ is $R_{s}=\sqrt{\frac{M_{s}}{0.16}} \mathrm{~cm}$, if the Hubble constant $H_{0}=71 \mathrm{~km} / \mathrm{sec} \mathrm{Mpc}$. In the holographic model, the number of sub-elements of mass $m$ in a large scale structure is $\frac{K}{m}$, where $K$ is constant, so the amount of information in any mass bin (proportional to $\frac{K}{m} m$ ) is the same in all mass bins. This is consistent with the $\frac{1}{m}$ behavior of the mass spectrum in the Press-Schechter formalism [4], and implies lowest mass sub-elements are the most numerous.

The main idea of this analysis is that matter inside a core radius much smaller than the holographic radius of a large scale structure is accumulated in a central black hole, where the core radius is the radius at which lowest mass sub-elements can exist without being disrupted and drawn into the central black hole.

The analysis assumes visible large scale structures develop within isothermal spherical halos of dark matter. 
So, the matter density distribution in large scale structures is $\rho(r)=\frac{a}{r^{2}}$, where $r$ is the distance from the center of the structure and $a$ is constant. The mass $M_{s}$ within the holographic radius $R_{s}$ is

$M_{s}=4 \pi \int_{0}^{R_{s}} \frac{a}{r^{2}} r^{2} \mathrm{~d} r=4 \pi a R_{s}$, requiring $a=\frac{M_{s}}{4 \pi R_{s}}$. Then, the mass within radius $R$ from the center of a large scale structure is $M_{R}=4 \pi \int_{0}^{R} \frac{a}{r^{2}} r^{2} \mathrm{~d} r=\frac{R}{R_{s}} M_{s}$ and the tangential speed $v_{t}$ of a sub-element of mass $m$ moving in a circle of radius $R$ around the center is found from $\frac{G M m}{R^{2}}=\frac{4 \pi G a m}{R}=\frac{m v_{t}^{2}}{R}$, where $G=6.67 \times 10^{-8}$ $\mathrm{cm}^{3} \cdot \mathrm{g}^{-1} \cdot \mathrm{sec}^{-2}$. So, the tangential speed of sub-elements in circular orbits around the center, $v_{t}=\sqrt{G \frac{M_{s}}{R_{s}}}$, does not depend on distance from the center and sub-elements lie on a flat tangential speed curve.

With an $\frac{a}{r^{2}}$ matter density distribution, sub-elements orbiting the center of a large scale structure at radius $R$ are equivalent to sub-elements orbiting a point mass with mass $\frac{R}{R_{s}} M_{s}$. In large scale structures with $a / r^{2}$ density distributions, the mass within a core radius $R_{c}$ is drawn into a central black hole with an innermost stable circular orbit (ISCO) radius [5] much less than the holographic radius of the lowest mass sub-element. The core radius $R_{c}$ is the holographic radius of the lowest mass sub-element of the large scale structure because no subelement can exist as an isolated system closer to the central black hole than the holographic radius of the lowest mass sub-element without being disrupted and drawn into the central black hole. In consequence, the upper bound on the mass concentrated in the central black hole of the large scale structure is the mass of the $a / r^{2}$ density distribution within $R_{c}$. The upper bound is reached when the central black hole has accumulated all of the matter within the central volume inside $R_{c}$. With density distribution $\rho(r)=\left(\frac{M_{s}}{4 \pi R_{s}}\right) \frac{1}{r^{2}}$, the mass of the structure within radius $R_{c}$ from the center of the large scale structure is $\frac{R_{c}}{R_{s}} M_{s}$. So, when the mass within the core radius $R_{c}$ is concentrated in the central black hole of the large scale structure, the upper bound on the mass of the central black hole is $\frac{R_{c}}{R_{s}} M_{s}$. The corresponding upper bound on the fraction of the mass of large scale structures concentrated in the central black hole is $\frac{R_{c}}{R_{s}}=\sqrt{\frac{M_{\min }}{M_{s}}}$, where $M_{\min }$ is the mass of the lowest mass sub-elements of the large scale structures.

Note that the innermost stable circular orbit (ISCO) radius, $r_{I S C O}$, of a black hole of mass $M_{\bullet}$. and spin angular momentum $J$ depends on its gravitational radius $r_{G}=\frac{G M}{c^{2}}$, where $c=3.00 \times 10^{10} \mathrm{~cm} \cdot \mathrm{sec}^{-1}$, and its spin $J$. The ISCO radius is $r_{I S C O}=6 r_{G}$ for a non-rotating black hole, $r_{I S C O}=r_{G}$ for maximal prograde rotation of the black hole and $r_{I S C O}=9 r_{G}$ for maximal retrograde rotation of the black hole [5]. So, the necessary condition for this analysis, $\frac{r_{I S C O}}{R_{c}} \ll 1$, is $\left(\frac{9 G}{c^{2}}\right)\left(0.16 R_{s}\right) \ll 1$. For the largest of all structures, with Jeans' mass $2.61 \times 10^{50}$ $\mathrm{g}$ and holographic radius $4.08 \times 10^{25} \mathrm{~cm}$,

$\left(\frac{9 G}{c^{2}}\right)\left(0.16 R_{s}\right)=0.0044$. Thus, central black holes are point particles compared to the holographic radius of the smallest sub-elements in any of the self-similar large scale structures.

\section{Central Black Holes at $z=0$}

This upper bound on the mass of central black holes in large scale structures is consistent with two important test cases. The first is the supermassive black hole in Sagittarius $A^{*}$ near the center of our Milky Way. The mass of the Milky Way is estimated as $2.52 \times 10^{45} \mathrm{~g}$ [6]. If the Hubble constant $H_{0}=71 \mathrm{~km} / \mathrm{sec} \mathrm{Mpc}$, the holographic model of self-similar large scale structure [2] estimates the mass of lowest mass star cluster sub-elements of galaxies as $1.0 \times 10^{35} \mathrm{~g}$. Then, the upper bound on the mass of the central black hole in the Milky Way is $1.6 \times 10^{40} \mathrm{~g}$, about twice the observed $9 \times 10^{39} \mathrm{~g}$ mass [7] of the supermassive black hole in Sagittarius $A^{*}$. The upper bound on the central black hole mass for galaxies with the average galactic mass [2] $1.6 \times 10^{44} \mathrm{~g}$ is $4.0 \times$ $10^{39} \mathrm{~g}$, or $2 \times 10^{6} M_{\odot}$, where $M_{\odot}=2 \times 10^{33} \mathrm{~g}$ is the solar mass.

In the holographic model for large scale structure, the constant relating the mass of isolated structures to their holographic radius, as well as the mass of lowest mass substructures within large scale structures, depends on the Hubble constant $H_{0}$. If the Hubble constant $H_{0}=65$ $\mathrm{km} / \mathrm{sec} \mathrm{Mpc}$, the upper bound on the mass of the central black hole in the Milky Way is $9.2 \times 10^{39} \mathrm{~g}$, close to the estimate from Keck telescope observations [7].

Self-similarity of large scale structures in the holographic model indicates there should be black holes in the centers of superclusters and star clusters, just as there are in galaxies. The largest black hole at $z=0$ should 
be in the center of the largest supercluster, corresponding to a supercluster with the Jeans' mass $2.6 \times 10^{50} \mathrm{~g}$. Using the estimate of $1.4 \times 10^{40} \mathrm{~g}$ for the lowest mass galaxies from the holographic model [2], the upper bound for the mass of the largest supermassive black hole in the universe at $z=0$ is $1.9 \times 10^{45} \mathrm{~g}$. This is about fifty times the $4.2 \times 10^{43} \mathrm{~g}$ mass of one of the largest black holes found to date, that in NGC 4889 in the Coma constellation [8].

At the lower end of the range of large scale structures, the holographic model estimates an average star cluster mass of $1.2 \times 10^{39} \mathrm{~g}\left(2 \times 10^{6} M_{\odot}\right)$ at $z=0$. Using a $z=0$ minimum stellar mass of $0.08 M_{\odot}=1.6 \times 10^{32} \mathrm{~g}, 4.4 \times$ $10^{35} \mathrm{~g}\left(220 M_{\odot}\right)$ is the upper bound on the central black hole in such a star cluster. The holographic upper bound on central black hole mass in star clusters is consistent with upper bounds on central black hole masses in globular clusters M15, M19 and M22 developed using the Jansky Very Large Array (JVLA) in New Mexico [9]. The mass of M15 is $5.6 \times 10^{5} M_{\odot}$ [10], the mass of M19 is $1.1 \times 10^{6} M_{\odot}$ [11], and the mass of M22 is

$2.9 \times 10^{5} M_{\odot}[10]$. The holographic upper bound for the mass of the central black hole in M15 of $212 M_{\odot}$, consistent with the JVLA upper bound of $980 M_{\odot}$. Correspondingly, the upper bound on the central black hole mass of $297 M_{\odot}$ for M19 is consistent with the JVLA upper bound of $730 M_{\odot}$, and the upper bound on the central black hole mass of $150 M_{\odot}$ for M22 is consistent with the JVLA upper bound of $360 M_{\odot}$.

\section{Supermassive Black Holes at $\mathrm{z}>0$}

In the holographic model [2], the range of the mass spectrum at any structural level decreases with redshift, because the mass at the lower end of the mass spectrum at any structural level increases with redshift. Also, the number of structural levels increases with redshift. Accordingly, for a given structure mass, the upper bound on central black hole mass increases with redshift. The holographic upper bound on central black hole mass for a structure with mass equal to that of the Milky Way is 6.1 $\times 10^{41} \mathrm{~g}$ at $z=0.5$ and $8.1 \times 10^{42} \mathrm{~g}$ at $z=1$, compared to the upper bound of $1.6 \times 10^{40} \mathrm{~g}$ at $z=0$. The estimated mass of an average galaxy at $z=0$ in the holographic model is $1.6 \times 10^{44} \mathrm{~g}$. The upper bound on the central black hole mass $M$. for a structure with mass $1.6 \times 10^{44}$ $\mathrm{g}$ is $1.5 \times 10^{41} \mathrm{~g}$ at $z=0.5$ and $2.1 \times 10^{42} \mathrm{~g}$ at $z=1$, compared to $4.0 \times 10^{39} \mathrm{~g}$ at $z=0$. This is consistent with indications that the average ratio $\frac{M_{\bullet}}{M_{\text {galaxy }}}$ increases with redshift [12].

Volonteri [1] says the "golden era" of 1 billion $M_{\odot}$ supermassive black holes "occurred early on." So, the analysis below estimates upper bounds on black hole masses in the early universe at $z>6$, less than a billion years after the end of inflation [13] and before development of self-similar large scale structures present in today's universe began at $z<6[2]$.

If the Hubble constant $H_{0}=71 \mathrm{~km} / \mathrm{sec} \mathrm{Mpc}$, the critical density $\rho_{\text {crit }}=\frac{3 H_{0}^{2}}{8 \pi G}=9.5 \times 10^{-30} \mathrm{~g} / \mathrm{cm}^{3}$. Assuming the universe is dominated by vacuum energy resulting from a cosmological constant $\Lambda$, matter accounts for about $26 \%$ of the energy in today's universe [14]. So, the matter density $\rho_{m}(z)$ at redshift $z$ is $\rho_{m}(z)=(1+z)^{3}$ $\rho_{m}(0)$, where today's matter density is $\rho_{m}(0)=0.26 \rho_{\text {crit }}=$ $2.5 \times 10^{-30} \mathrm{~g} / \mathrm{cm}^{3}$. Correspondingly, the cosmic microwave background radiation density at redshift $z$ is $\rho_{r}(z)=$ $(1+z)^{4} \rho_{r}(0)$, where the mass equivalent of today's radiation energy density is $\rho_{r}(0)=4.4 \times 10^{-34} \mathrm{~g} / \mathrm{cm}^{3}[15]$. When matter dominates, the speed of pressure waves affecting matter density at redshift $z$ is

$c_{s}(z)=c \sqrt{\frac{4(1+z) \rho_{r}(0)}{9 \rho_{m}(0)}}[16]$, and the Jeans' length $L(z)=c_{s}(z) \sqrt{\frac{\pi}{G(1+z)^{3} \rho_{m}(0)}}[16,17]$. The first level of large scale structure within the universe is determined by the Jeans' mass $M(z)=\frac{4 \pi}{3}\left(\frac{L(z)}{4}\right)^{3} \rho_{m}(z)$, where $L(z)=\frac{(1+z)^{2}}{\rho_{m}(z)} \frac{2 c}{3} \sqrt{\frac{\pi \rho_{r}(0)}{G}}=\frac{(1+z)^{2} B}{\rho_{m}(z)}$, and $B=\frac{2 c}{3} \sqrt{\frac{\pi \rho_{r}(0)}{G}}=2.89 \times 10^{-3} \mathrm{~g} / \mathrm{cm}^{2}$. So the Jeans' mass $M(z)=\frac{\pi B^{3}}{48 \rho_{m}^{2}(0)}$ is independent of $z$ [16].

Consider the era at $z>6$, before self-similar large scale structure developed [2], when each Jeans' mass was populated by early stars, with masses in the range $2 \times$ $10^{34} \mathrm{~g}$ to $2 \times 10^{35} \mathrm{~g}\left(10 M_{\odot}\right.$ to $\left.100 M_{\odot}\right)$ [18]. Taking this range as bounds on the lowest mass of early stellar systems, the holographic radii of the lowest mass early stellar systems, and thus the central core radii $R_{c}$ of the matter distribution within the Jeans' masses, was between $3.6 \times 10^{17} \mathrm{~cm}$ and $1.1 \times 10^{18} \mathrm{~cm}$. Then, the analysis above indicates each Jeans' mass should harbor a central supermassive black hole with upper bounds on its mass in the range $2.3 \times 10^{42} \mathrm{~g}$ to $7.2 \times 10^{42} \mathrm{~g}$. This estimate is consistent with observation [19] of the $4 \times 10^{42} \mathrm{~g}$ black hole in the quasar ULAS J112001.48 + 064124.3 at redshift $z=7.085$. If the lowest mass of early stars was $30 M_{\odot}$, the upper bound of $4 \times 10^{42} \mathrm{~g}$ on the mass of black holes in the center of each Jeans' mass equals the mass observed in ULAS J112001.48 + 064124.3. Later, 
at $z>6$, as self-similar large scale structure developed, supermassive black holes formed within lower structural levels, and almost all systems composed of stars or star aggregations developed central supermassive black holes.

\section{Central Black Hole Development}

Development of visible large scale structures within isothermal spherical halos of dark matter with $a / r^{2}$ density distributions resulted in the commonly observed flat tangential speed distribution of sub-elements. In the holographic model of large scale structure, black holes near the center of nascent large scale structures are progenitors of supermassive black holes. Any sub-element passing within a distance from the central black hole that is less than the holographic radius of the sub-element is disrupted and drawn into the central black hole. So, the mass within a core radius $R_{c}$ is drawn into in the central black hole. The core radius $R_{c}$ is the holographic radius of the lowest mass sub-element of the large scale structure because no sub-element can exist as an isolated system closer to the central black hole than the holographic radius of the lowest mass sub-element without being disrupted and drawn into the central black hole. In consequence, the upper bound on the mass of the central black hole within $R_{c}$ in the $\rho(r)=\left(\frac{M_{s}}{4 \pi R_{s}}\right) \frac{1}{r^{2}}$ density distribution of the large scale structure is $\frac{R_{c}}{R_{s}} M_{s}$. Again, the upper bound is reached when the central black hole has accumulated all of the matter within the central volume inside $R_{c}$.

Stars with masses $>100 M_{\odot}$ developed at $z>10$. They had very short lives and many of them collapsed to black holes [20]. It has been claimed that black holes resulting from collapse of stars in the $100 M_{\odot}$ range might not suffice as seeds for supermassive black holes, so supermassive stars in the $10^{5} M_{\odot}$ range should be considered as seeds for supermassive black holes [21]. That scenario is consistent with the holographic model for large scale structure [2]. When photon decoupling took place, at $z \approx 1100$, "hydrogen gas was free to collapse under its own self-gravity (and the added gravitational attraction of the dark matter)" [22]. The extended holographic principle used in the holographic model of large scale structure [2] indicates the information describing a structure of mass $M_{s}$ is encoded on a holographic screen with radius $R_{s}=\sqrt{\frac{M_{s}}{0.16}} \mathrm{~cm}$, if the Hubble constant $H_{0}=71 \mathrm{~km} / \mathrm{sec}$ Mpc. Consider the escape velocity of protons on the holographic screen for a mass $M_{s}$ with radius $R_{s}$ at $z=1100$, and set it equal to the average velocity of protons in equilibrium with $\mathrm{CMB}$ radiation outside the screen. Then the holographic model for large scale structure [2] identifies $10^{5} M_{\odot}$ as the mass of systems in thermal equilibrium with the CMB, since there is no heat transfer between a system with mass $M_{s}=10^{5} M_{\odot}$ and the $\mathrm{CMB}$ at $z=1100$. At $z=$ 1100 , protons outside the holographic screen with radius $R_{s}=\sqrt{\frac{M_{s}}{0.16}} \mathrm{~cm}$ that are in equilibrium with the $\mathrm{CMB}$ cannot transfer heat (and energy) across the holographic screen surrounding a system with mass $M_{s}=10^{5} M_{\odot}$ at $z=1100$. The free fall time [23] for systems with mass $M_{s}=10^{5} M_{\odot}$ with the matter density at $z=1100$ is about 2.6 million years, so there is sufficient time for those systems to ignite as supermassive stars and subsequently collapse to seed black holes with masses near $10^{5} M_{\odot}$ [24] leading to formation of supermassive black holes in the 800 million years before emissions associated with the $4 \times 10^{42} \mathrm{~g}$ black hole in the quasar ULAS J112001.48 + 064124.3 observed at redshift $z=7.085$ [19]. Anyway, the first stars apparently produced seed black holes for subsequent development of large scale structures.

In the earliest phase of development of large scale structure, at $z \approx 6$, there was only one Jeans' mass structure level in the holographic model for large scale structure [2]. These earliest large scale structures, home of the earliest quasars, then developed around a seed black hole near their center. Sub-elements of the earliest large scale structures were early stars with masses in the $10 M_{\odot}$ to $100 M_{\odot}$ range, resulting in the estimated mass for supermassive black holes in the $z>6$ range mentioned above. As additional self-similar large scale structure levels developed, remaining seed black holes at the center of each emerging large scale structure grew by disrupting and entraining lowest mass sub-elements of the self-similar large scale structure.

\section{REFERENCES}

[1] M. Volonteri, Science, Vol. 337, 2012, pp. 544-547. doi:10.1126/science. 1220843

[2] T. Mongan, Journal of Modern Physics, Vol. 2, 2011, pp. 1544-1549. doi:10.4236/jmp.2011.212187

[3] R. Bousso, "The Holographic Principle," Reviews of Modern Physics, Vol. 74, 2002, pp. 825-874. doi:10.1103/RevModPhys.74.825

[4] W. Press and P. Schechter, Astrophysical Journal, Vol. 187, 1974, pp. 425-438. doi:10.1086/152650

[5] M. Abramowicz and P. Fragile, "Foundations of Black Hole Accretion Disk Theory," 2011, arXiv:1104.5499.

[6] P. McMillan, "Mass Models of the Milky Way," 2011, arXiv:1102.4340.

[7] A. Ghez, et al., "Measuring Distance and Properties of 
the Milky Way's Central Supermassive Black Hole with Stellar Orbits," 2008, arXiv:0808.2870.

[8] N. McConnell, et al., Nature, Vol. 480, 2011, pp. 215218. doi:10.1038/nature10636

[9] J. Strader, et al., Astrophysical Journal Letters, Vol. 750, 2012, p. L27. doi:10.1088/2041-8205/750/2/L27

[10] M. Marks and P. Kroupa, Monthly Notices of the Royal Astronomical Society, Vol. 406, 2010, p. 2000.

[11] J. Boyles, et al., Astrophysical Journal, Vol. 742, 2011, p. 51. doi:10.1088/0004-637X/742/1/51

[12] C. Booth and J. Schaye, Monthly Notices of the Royal Astronomical Society, Vol. 412, 2011, pp. 1158-1164. doi:10.1111/j.1365-2966.2011.18203.x

[13] E. Wright, Astronomical Society of the Pacific, Vol. 118, 2006, p. 1711. doi: $10.1086 / 510102$

[14] W. Percival, et al., Astrophysical Journal, Vol. 657, 2007, p. 51. doi:10.1086/510772

[15] A. Siemiginowska, et al., Astrophysical Journal, Vol. 657, 2007, p. 145. doi:10.1086/510898

[16] S. Longair, "Galaxy Formation," Springer-Verlag, Berlin, 1998. doi:10.1007/978-3-662-03571-9
[17] J. Jeans, Philosophical Transactions of the Royal Society of London, Vol. 199, 1902, pp. 1-53.

[18] V. Bromm, "The First Stars and Galaxies-Basic Principles," In M. De Rossi, et al., Eds., From the First Structures to the Universe Today, 2012, arXiv:1203.3824.

[19] D. Mortlock, et al., Nature, Vol. 474, 2011, p. 616. doi:10.1038/nature10159

[20] V. Bromm and R. Larson, Annual Review of Astronomy and Astrophysics, Vol. 42, 2004, pp. 79-118. doi:10.1146/annurev.astro.42.053102.134034

[21] J. Johnson, et al., "Supermassive Seeds for Supermassive Black Holes,” 2012, arXiv:1211.0548.

[22] B. Ryden, "Introduction to Cosmology," Addison-Wesley, San Francisco, 2003, p. 161

[23] S. Hofner, "Gravitational Collapse: Jeans Criterion and Free Fall Time," 2012.

http://www.astro.uu.se/ hoefner/astro/teach/apd_files/apd _collapse.pdf

[24] S. Shapiro and M. Shibata, Astrophysical Journal, Vol. 577, 2002, p. 904. doi:10.1086/342246 